OCCASIONAL VIEWPOINT

\title{
Creutzfeldt-Jakob disease: implications for gastroenterology
}

\author{
M G Bramble, J W Ironside
}

Gut 2002;50:888-890

The current clinical views regarding variant Creutzfeldt-Jakob disease, and in particular transmission via endoscopy, of those representing both gastroenterology and the Spongiform Encephalopathy Advisory Committee are presented in an attempt to guide clinicians as to "best practice" given the current state of our knowledge.

See end of article for authors' affiliations

Correspondence to Professor MG Bramble, Endoscopy Centre, James Cook University Hospital, Marton Rd, Middlesbrough TS4 3BN, UK: mike.bramble@stees.nhs.uk

Accepted for publication 19 November 2001
M ost gastroenterologists working in the UK have been aware for some time that endoscopy may be a vector for the transmission of prions from a patient incubating, but not clinically manifesting, variant Creutzfeldt-Jakob disease (vCJD) to the next individuals undergoing the same procedure on the same list. To date there are no recorded cases of iatrogenic transmission of VCJD via endoscopy but it remains a risk which will be present for many years to come. Advice to health authorities on individual cases is through the CJD Incidents Panel. However, we are aware that advice to health professionals performing endoscopy needs to be as comprehensive as current evidence will allow, without making it impossible to perform endoscopic procedures on patients who will clearly derive long term health benefits from an accurate endoscopic diagnosis and/or treatment. This article represents the current clinical views of those representing both gastroenterology and the Spongiform Encephalopathy Advisory Committee (SEAC). Both authors sit on the CJD Incidents Panel and have been advising the Department of Health on individual cases during the last year. It is important to note that the advice given in this article may be superseded if additional information or evidence becomes available.

CJD is a member of a group of neurological disorders known as the transmissible spongiform encephalopathies or prion diseases, which affect both animals (such as scrapie in sheep or bovine spongiform encephalopathy (BSE) in cows) and humans. The precise nature of the transmissible agents responsible for these disorders is unknown but there is increasing evidence to support the prion hypothesis, which states that the agent is composed of an abnormally folded form of a host encoded protein, prion protein. The normal prion protein $\left(\mathrm{PrP}^{\mathrm{c}}\right)$ is expressed in many tissues but occurs at the highest levels in neurones in the central nervous system (CNS) where it may act as a copper binding protein, although its precise physiological role is unknown. The abnormal form of the protein $\left(\mathrm{PrP}^{\mathrm{sc}}\right)$ accumulates in the CNS in prion diseases; the infectious agent is remarkably resistant to most forms of degradation. The association between $\operatorname{PrP}^{\mathrm{Sc}}$ and the gut has been eloquently described in a previous leading article ${ }^{1}$ and gastroenterologists need to understand where we are in terms of our present day knowledge of this entity.

In humans, prion diseases occur in three major categories: sporadic, acquired, and familial. All are currently untreatable and universally fatal although recent studies have indicated that a combination of drugs may be effective in experimental prion diseases ${ }^{2}$ : this approach is under consideration as a clinical trial. The sporadic form of CJD affects approximately one person per million per annum in the population on a worldwide basis. CJD has also occurred as an acquired iatrogenic disorder, transmitted to other humans through direct (inadvertent) inoculation of the brain via contaminated neurosurgical instruments, via corneal and dura mater grafts, or through administration of human pituitary extracts used to treat growth hormone or gonadotrophin deficiency. Variant CJD (vCJD) is a new acquired form of CJD which was first reported in 1996 affecting mainly young adults and with a unique neuropathological phenotype. ${ }^{3}$ It is now widely accepted that bovine prions passed into the human population through consumption of BSE infected bovine tissues; the transmissible agent responsible for vCJD is identical to the BSE agent (but different from the agent in sporadic CJD). The incubation period for vCJD is likely to be lengthy and may have a mean value of 10-30 years. During this time the affected person has the potential to transmit the disease to others via surgical procedures which might result in the transfer of infected tissue into the next person operated on with the same surgical instruments.

The distribution of $\operatorname{PrP}^{\mathrm{sc}}$ in the body is different in sporadic and variant CJD, reflecting the different pathogenesis of the two forms. In the case of sporadic CJD, prion infectivity is largely limited to the CNS (including the retina) and only operations involving the brain and eye have resulted in iatrogenic transmission of the disease. Gastrointestinal endoscopy is unlikely to be a vector for the transmission of sporadic CJD as infected tissue is not encountered during the procedure. No special precautions are necessary during or after the procedure and the endoscope should be cleaned and disinfected in the normal thorough way. ${ }^{4}$

Abbreviations: vCJD, variant Creutzfeldt-Jakob disease; BSE, bovine spongiform encephalopathy; PrP, prion protein; CNS, central nervous system. 
"Endoscopy on patients who are incubating vCJD may result in exposure of the instrument (and particularly the biopsy forceps) to PrPsc"

In contrast, in vCJD the lymphoreticular system throughout the body contains $\mathrm{PrP}^{\mathrm{sc}}$ at the time of death, and experimental evidence suggests that the lymphoreticular system may contain significant levels of infectivity for most of the incubation period. ${ }^{5}$ To support this, in vCJD abnormal prion protein was found in the germinal centres in the wall of an appendix from a vCJD patient that was removed eight months before the onset of neurological disease. ${ }^{6}$ As lymphoid follicles and germinal centres are widely distributed in the gastrointestinal tract (and are often biopsied), it is possible that endoscopy on patients who are incubating VCJD may result in exposure of the instrument (and particularly the biopsy forceps) to $\mathrm{PrP}^{\mathrm{sc}}$. Consequently, the question now arises, how great is the risk of secondary (person to person) transmission in endoscoping a patient incubating vCJD? There are three scenarios which gastroenterologists are likely to encounter and this editorial will attempt to guide clinicians as to "best practice" given the current state of our knowledge.

\section{UPPER GASTROINTESTINAL ENDOSCOPY \\ Scenario No 1}

Occasionally gastroenterologists may be requested to endoscope a patient with known or probable sporadic CJD (usually to site a PEG feeding tube). This can be carried out in the routine way provided VCJD is not suspected. If inadvertently a patient with suspected vCJD is endoscoped, the instrument used should be quarantined until the postmortem diagnosis is known. If sporadic CJD is diagnosed, the endoscope can be returned to use following thorough cleaning and decontamination, as is normal practice. If VCJD is diagnosed the endoscope cannot be used again and should be quarantined or sent to the National CJD Surveillance Unit in Edinburgh for research purposes. The previous advice to destroy such instruments represents a lost opportunity to study the risks involved in more detail. It would also be good practice to inform colleagues locally that a quarantined instrument was available for use in other endoscopy units if they too had a patient with suspected vCJD requiring endoscopy.

\section{Scenario No 2}

For patients with known or probable vCJD, ${ }^{7}$ endoscopy should only be a last resort. Ultrasound guided insertion of a gastrostomy feeding tube would be preferable to a PEG feeding tube if local expertise is available. If not, endoscopy should be performed using an instrument already set aside for such patients. If no such instrument is available locally, one can be loaned to any hospital by the National CJD Surveillance Unit in Edinburgh (contact telephone number 0131537 1980). If scenario No 2 becomes more common, endoscopes may need to be held regionally for this purpose.

\section{Scenario No 3}

This scenario covers patients who have been endoscoped by an instrument previously used on a patient who was not known to be incubating vCJD at the time of endoscopy but who subsequently went on to develop the disease. This could become the commonest scenario and it must be assumed that the patient who went on to develop vCJD was incubating the disease at the time of the original endoscopy. This also means that infectious material may not have been removed completely by current methods of decontaminating endoscopes, and that subsequent patients have been exposed to the prion agent. The instrument used should therefore be quarantined until advice has been sought from the CJD Incidents Panel (Department of Health, Skipton House, London; contact telephone 02079721761 ) as to the management of the situation. Local infection control teams will need to be involved with contact tracing and information handling.

\section{LOWER GASTROINTESTINAL ENDOSCOPY}

It is unlikely that colonoscopy would be clinically justifiable in a patient known or strongly suspected as suffering from vCJD. However, it is quite possible that an asymptomatic patient incubating VCJD may undergo colonoscopy prior to diagnosis and this situation is essentially the same as in scenario 3. The risks of transmitting prion protein to the next patient are much greater however, due to a number of factors which relate to the amount of lymphatic tissue encountered during endoscopy and the number, site, and size of mucosal biopsies obtained by this method.

In general the risks of transmitting vCJD from one patient to another are dependent on the infectivity of the tissues involved, the amount of tissue contaminating the instrument, the effectiveness of the decontamination processes, and the susceptibility of subsequently exposed patients. Experimental studies suggest that levels of infectivity in prion diseases are highest in the CNS and retina, which are approximately two logs higher than in the tonsils and other lymphoreticular tissue. A recent study has also detected the abnormal form of the prion protein in rectal tissue from a patient with vCJD by western blot examination of autopsy tissues. ${ }^{8}$ The risk of transmitting vCJD through the endoscopy procedure itself is likely to be small, but contamination of the endoscope and forceps as a result of biopsy of lymphoid tissues may represent a larger (but currently unquantifiable) risk, even though only small amounts of tissue are involved.

"The risks of transmitting vCJD from one patient to another are dependent on the infectivity of the tissues involved, the amount of tissue contaminating the instrument, the effectiveness of the decontamination processes, and the susceptibility of subsequently exposed patients"

The greatest risk is undoubtedly that which ensues from biopsy of the terminal ileum where Peyer's patches may contain significant levels of prion protein for a patient incubating vCJD. The biopsy forceps and the colonoscope become potential vectors for disease transmission under these circumstances. Meticulous manual cleaning of the colonoscope is probably the best defence against person to person transmission. The same is true of the biopsy forceps, but as disposable forceps are now available there is a strong argument for moving towards the universal use of disposable biopsy forceps for mucosal samples taken at colonoscopy. Endoscopy units should now work towards a policy of using disposable biopsy forceps as the only practical way of minimising the risk which results from ileal biopsy. In addition, "random" biopsies should be kept to a minimum as lymphoid tissue is distributed widely throughout the gastrointestinal tract. Although thorough cleaning of flexible endoscopes ensures patient safety for "normal" pathogens, the same process may not be adequate for the $\operatorname{PrP}^{\mathrm{sc}}$. The main benefit of the decontamination process under these circumstances is undoubtedly effective manual cleaning, as glutaraldehyde may stabilise $\mathrm{PrP}^{\mathrm{sc}}$ on the metal surface of the endoscope, with potentially adverse consequences. It follows that brushes used to clean the channels of the endoscope are used only once to ensure maximum efficiency and biopsy forceps should also be functioning optimally and discarded as soon as they appear to be under performing (tearing tissue rather than cutting it). The rubber valve protecting the biopsy channel is another item which is potentially disposable and serious consideration should be given to single use valves. Again, more research is required to 
determine "best practice". For rigid endoscopes, autoclaving at the recommended conditions for $\mathrm{CJD}^{9}$ is the best way of attempting decontamination.

What should endoscopists do in the short term? The answer to this question must be to ensure as far as possible that manual cleaning of endoscopes and reuseable accessories is of the highest standard. Endoscopy has a major role in patient care, and this should not be compromised unless it is absolutely unavoidable in the public interest. It is also essential that endoscopes should be individually identifiable and their use traceable in any given patient population. Random biopsies should be kept to an absolute minimum (particularly of the ileum in colonoscopy) and endoscopy itself should be as atraumatic as possible, especially gastroscopy where the instrument is in contact with the mucosa covering the tonsils. Biopsy forceps should be treated as "high risk" and undergo thorough ultrasonic cleaning followed by autoclaving. As research in the UK progresses, it is likely that other procedures will be developed to inactivate prion infectivity and to remove proteins from instrument surfaces. The development of such techniques (along with more sensitive tests for prion detection) may well have an impact on future advice concerning endoscopy and CJD.

Depending on the final numbers of people infected with vCJD, we must assume that a significant number may undergo endoscopy before neurological symptoms appear. ${ }^{10}$ It is therefore up to every endoscopist to be aware of the dangers and follow the advice set out here. Further advice on specific cases and possible exposure incidents can be obtained from the CJD Incidents Panel (Department of Health, Skipton House, London; contact telephone 0207972 1761).

\section{Authors' affiliations}

M G Bramble, Endoscopy Centre, James Cook University Hospital, Marton Road, Middlesbrough TS4 3BW, UK

J W Ironside, CJD Surveillance Unit, Western General Hospital, Crewe Road, Edinburgh EH4 2XU, UK

\section{REFERENCES}

1 Shmakov AN, Ghosh S. Prion proteins and the gut: une liaison dangereuse? Gut 2001;48:443-7.

2 Korth C, May BCH, Cohen FE, et al. Acridine and phenothiazine derivatives as pharmacotherapeutics for prion disease. Proc Natl Acad Sci USA 2001;98:9836-41.

3 Will RG, Ironside JW, Zeidler $M$, et al. A new variant of Creutzfeldt-Jakob disease in the UK. Lancet 1996:347:921-5.

4 Report of a Working Party of the British Society of Gastroenterology Endoscopy Committee. Cleaning and disinfection of equipment for gastrointestinal endoscopy. Gut 1998;42:585-93.

5 Hill AF, Butterworth RJ, Joiner $S$, et al. Investigation of variant Creutzfeldt-Jacob disease and other human prion diseases with tonsil biopsy samples. Lancet 1999;353:183-9.

6 Hilton DA, Fathers E, Edwards P, et al. Prion immunoreactivity in the appendix before the clinical onset of new variant Creutzfeldt-Jacob disease. Lancet 1998:352:703-4.

7 Will RG, Zeidler M, Stewart GE, et al. Diagnosis of new variant Creutzfeldt-Jakob disease. Ann Neurol 2000;47:575-82

8 Wadsworth JD, Joiner S, Hill AF, et al. Tissue distribution of protease resistant prion protein in variant Creutxfleldt-Jakob disease using a highly sensitive immunoblotting assay. Lancet 2001;358:171-80.

9 Dangerous Pathogens Spongiform Encephalopathy Advisory Committee. Transmissible spongiform encephalopathy agents: safe working and the prevention of infection. London: The Stationary Office, 1998.

10 Ironside JW, Hilton DA, Ghani A, et al. Retrospective study of prion protein accumulation in tonsil and appendix tissue. Lancet 2000;355: 1693-94. 